\title{
The MCP-based system for monitoring space-time characteristics of the Nuclotron circulating beam
}

\section{Anton Baldin}

JINR

Dubna, Russia

E-mail: an.baldin@mail.ru

\section{Anatoliy Berlev}

JINR

Dubna, Russia

E-mail: berlev@inr.ru

\section{Andrey Fedorov}

JINR

Dubna, Russia

E-mail: andrei.fedorov.60@gmail.com

\section{Ivan Kudashkin ${ }^{1}$}

JINR

Dubna, Russia

E-mail: ivan.kudashkin@gmail.com

The advanced diagnostic system for the Nuclotron circulating beams based on microchannel plates (MCP) is presented in the framework of the NICA project and experiments with extracted beams. The system, designed, developed and tested on the beams during the four last runs of the Nuclotron, provides measurements in the intensity range of $10^{5}-10^{9}$ for singly charged ions which is not covered by existing measuring devices.

XXI International Baldin Seminar on High Energy Physics Problems

September 10-15, 2012

JINR, Dubna, Russia

\footnotetext{
${ }^{1}$ Speaker
} 


\section{Introduction}

Construction of modern nondestructive control systems of space-time characteristics of the beam during acceleration and extraction is one of the most important tasks for the Nuclotron accelerator complex (JINR LHEP).

Since the Nuclotron is planned to accelerate ions of a wide range up to $\mathrm{Au}$; there is a problem of adjustment and control of various beams extraction especially at low intensity (from units to $10^{6}$ particles per acceleration cycle). The construction of diagnostic systems for lowintensity beams is also required in applied research: radiation resistance of electronic components, radiobiological research, etc.

At present, the beam position in the Nuclotron accelerator chamber is measured by pickup electrodes but detectors of this type are insufficiently sensitive at low beam intensities (less than $10^{8}$ singly charged particles circulating in the accelerator chamber).

For this purpose there are also detectors based on SEM (Secondary Electron Multiplier) but these detectors do not allow one to control the spatial characteristics of the Nuclotron beam because the entrance window of the SEM is too big $(10 \mathrm{~mm})$ for the cross-sectional dimensions of the Nuclotron beam.

The developed MCP-based system for the circulating beam monitoring is nondestructive, possesses sufficient sensitivity and space-time resolution.

\section{Principle of operation}

The idea of the method to measure space-time beam characteristics is as follows: an accelerated particle beam ionizes residual gas in a vacuum chamber, and the MCP-based detector embedded beyond the orbit of the circulating beam, detects these ions.

A microchannel plate is sensitive to single charged particles hitting its surface. The amplification of a chevron assembly consisting of two plates is from $10^{4}$ to $10^{6}[2,3]$. The ion detection efficiency of MCP-based detectors is close to $100 \%$ and the time resolution is about 100 ps. Detectors of this type allow one to perform a multidimensional space-time analysis of beam distribution during its acceleration and extraction [1].

MCP detectors possess radiation resistance that allows one to use them in accelerator technology $[5,6]$.

The disadvantage of the detectors of this type is a long recovery time of the MCP area (group of activated channels) at the point hit by the registered ion. Since each channel operates as a photomultiplier, the charge depletion of activated channels providing the signal amplification takes place. For a number of channels equal to $10^{6} \mathrm{~cm}^{-2}$ the loading capacity of the MCP-based detector can be estimated. The plate recovery time after overload is about $100 \mathrm{~ms}$.

Our new development of the beam monitoring system is based on the results of the activity described in [4].

The detector consists of two MCPs (chevron assembly) located at a distance of $250 \mu \mathrm{m}$. The plate size is $43 \times 63 \mathrm{~mm}$, the channel diameter is $15 \mu \mathrm{m}$. The contact areas (pads) electrically isolated from each other are used as the collector of electron avalanches. The voltage is supplied via a passive splitter to each side of the MCP along its perimeter. Then the amplified signals are 
recorded from 16 pads of the printed circuit board placed under the MCP chevron assembly. The pads were grounded via $3 \mathrm{k} \Omega$ resistors for draining the accumulated charge.

\section{Testing and adjustment of the system}

A specialized vacuum test bench was designed and manufactured. The $\alpha$ source was used for testing at the ion energy of $4 \mathrm{MeV}$. It was located above the assembly at a distance of $4 \mathrm{~cm}$ inside the vacuum chamber.

The following signal characteristics have been studied: the amplitude increases by 10 times if the voltage changes from -2000 to $-2550 \mathrm{~V}$; the signal width at the base is from 5 to $7 \mathrm{~ns}$ and practically does not depend on the plate voltage. For all the measured voltages the signal front is less than $1 \mathrm{~ns}$ providing the time resolution of the detector of $100 \mathrm{ps}$. The detector signal at supply voltage of $-2400 \mathrm{~V}$ is shown in Fig. 1.

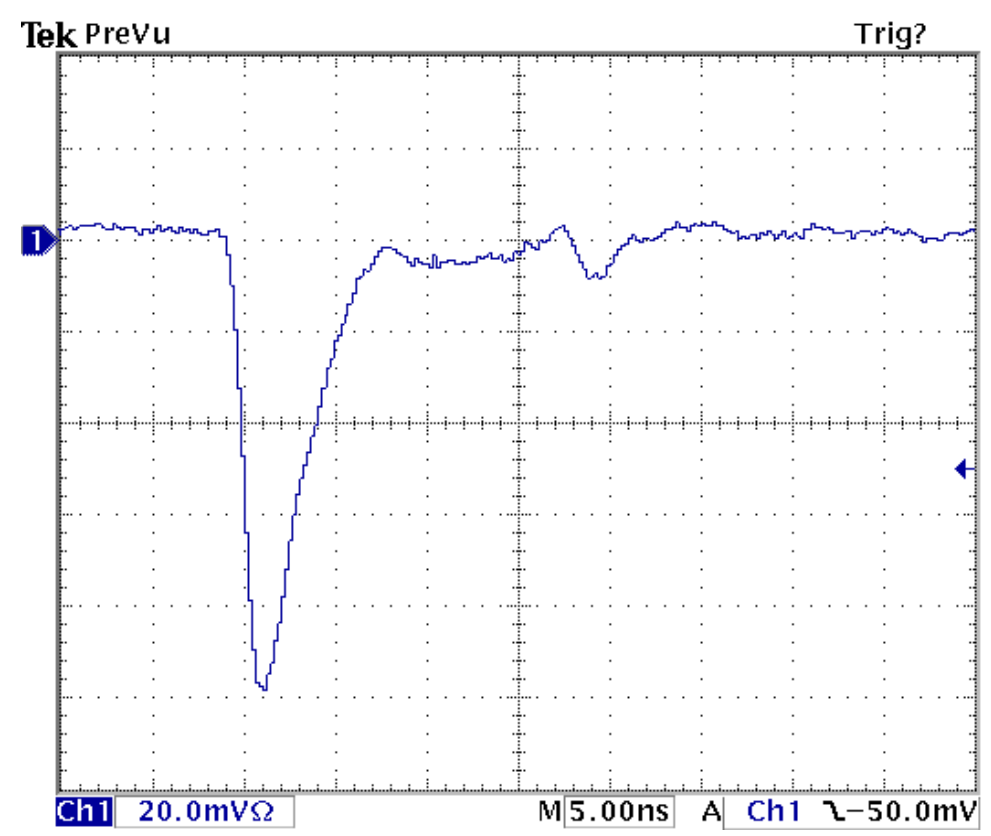

Figure 1: The detector signal recorded by using an oscilloscope. The detector supply voltage is $-2400 \mathrm{~V}$.

Taking into account the problem of recovery time of microchannel plates, the integral spectra of single signals have been studied depending on the supply voltage of the chevron assembly by using a charge-sensitive converter (Fig. 2). 


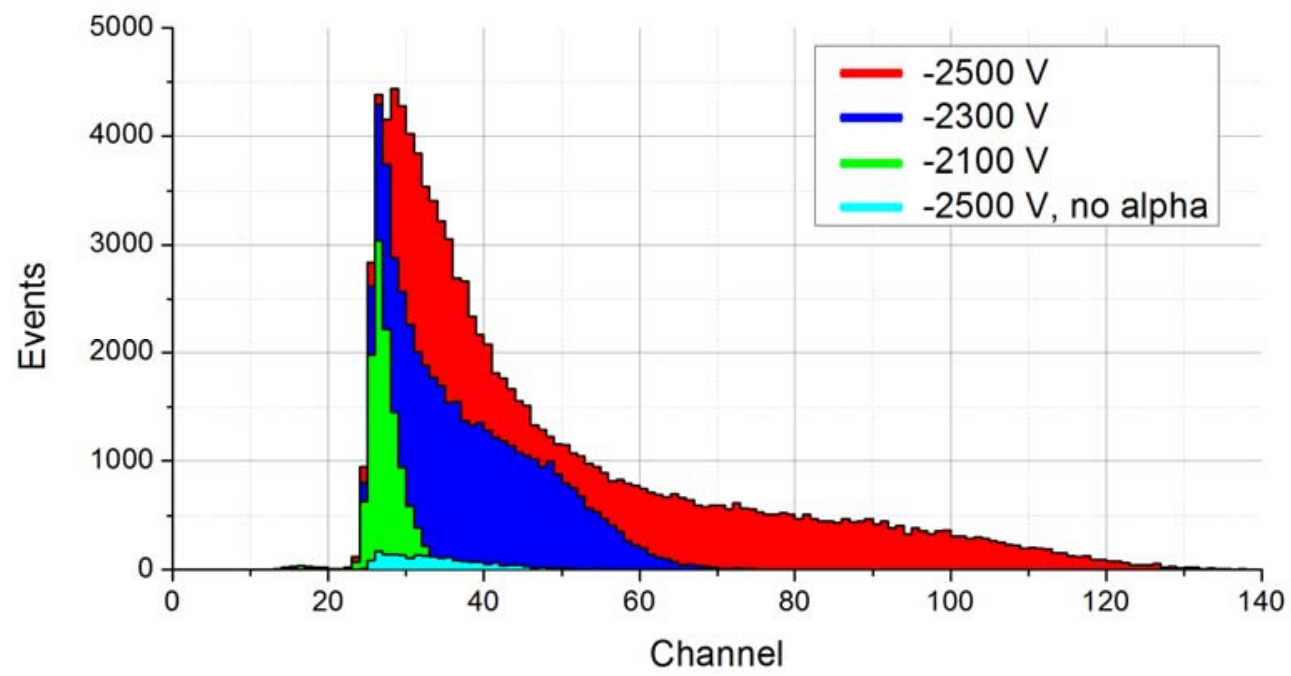

Figure 2: The histogram of the signal area (QDC spectra) for different supply voltage of the MCP assembly. The collecting time of each spectrum is $15 \mathrm{~min}$.

The signal amplification at ion interactions with the MCP grows with voltage increasing, and the charge and number of events above the discriminator threshold also increase (the threshold was $-20 \mathrm{mV}$ in this experiment). The measurements without an $\alpha$ source and the maximum supply voltage of $-2500 \mathrm{~V}$ (Fig. 2) were carried out to determine the noise spectrum. The number of events in the noise component is negligible, as compared to the signal from the $\alpha$ source.

The detector was tested at the vacuum test bench under the conditions quite different from those in the accelerator chamber. The vacuum conditions and the particle flow hitting the detector differ by orders of magnitude. The number of ions incident on the detector per time unit is higher by three orders of magnitude than that from the $\alpha$ source at the test bench experiment.

In 2010 the detector was installed into the accelerator ring and worked during the run. The aim of those measurements was to study realistic operation conditions for existing density of residual gas in the accelerator chamber and typical intensities of the accelerated beams. The load capacity of the detector was determined: the recovery time of the plate was about $100 \mathrm{~ms}$; the maximum number of ions registered by the detector without overloading was $2 \cdot 10^{4}\left[\right.$ ions $\left./\left(\mathrm{s} \cdot \mathrm{cm}^{2}\right)\right]$.

The detector overloading was the main problem during its testing on the beam. The detector operated at low beam intensities (less than $1 \cdot 10^{8}$ ) only. For this reason a new detector was developed to register the space-time characteristics of the radial beam component (Fig. 3). 


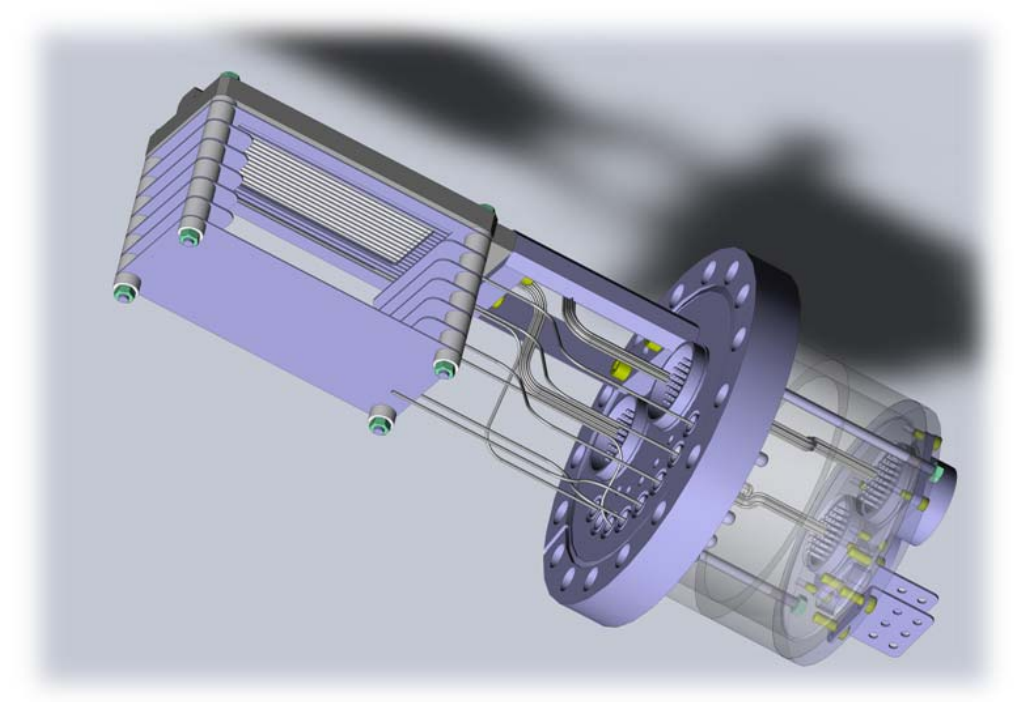

Figure 3: The design of the new detector for diagnostics of the beem radial coordinate.

The detector uses microchannel plates produced by "Baspik" company (Russia). The plate size is $96 \times 33 \mathrm{~mm}$, the channel diameter is $15 \mu \mathrm{m}$. Totally 32 pads positioned with a step of $3 \mathrm{~mm}$ are used as the collector of electron avalanches. The system of separate high voltage supply of each MCP is used to eliminate overloading. An additional cutoff mesh electrode was installed at a distance of $8 \mathrm{~mm}$ from the detector plane to control the ion flow (Fig. 4). The new system to register the beam coordinate possesses a higher spatial resolution and can operate with intensities up to $10^{9}$ particles per acceleration cycle.

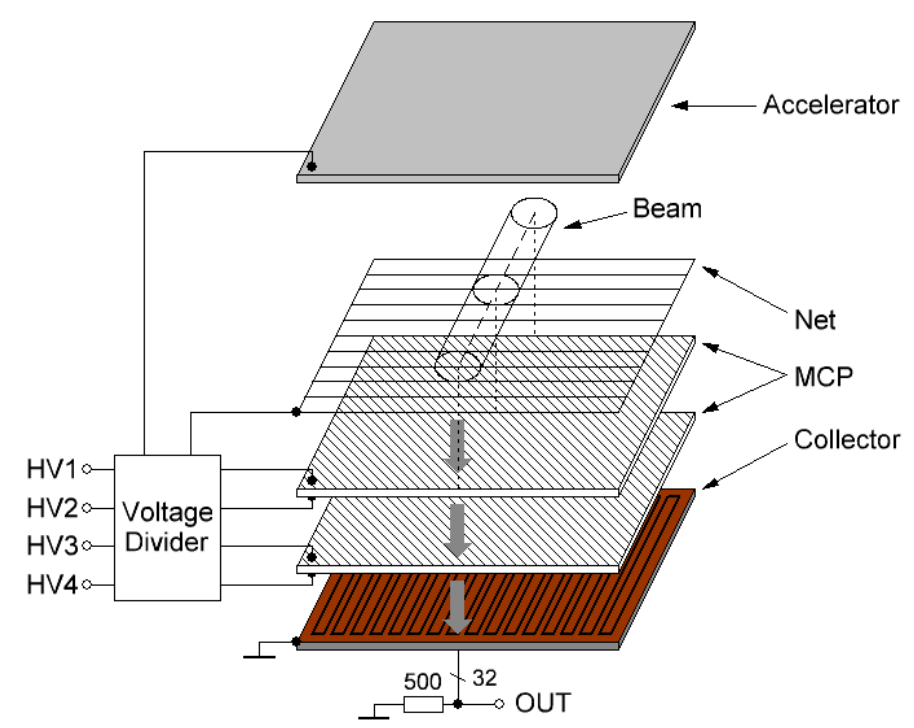

Figure 4: The MCP-based system for measuring the radial coordinate of the Nuclotron beam.

The specialized electronic module and software for remote control, visualization and data recording were developed to provide the detector operation in the accelerator ring. The electronic module has 32 channels. Each channel is supplied with an analog signal from the 
MCP detector and has a discriminator with a computer-controlled threshold. If the amplitude of the analog signal at the input exceeds the discriminator threshold, a digital signal is produced at the output. The module counts the number of pulses per time interval and records this number as a hexadecimal word into the module memory. The interval is set in the program and makes from $50 \mathrm{~ns}$ to $40 \mathrm{~ms}$. The module sequentially processes 1024 of these intervals and counts the number of signals exceeding the set threshold in each interval for each channel of the detector. So, the matrix of $32 \times 1024$ positive integer numbers is obtained at the output.

The control program of the electronic module displays online the beam distribution in the vacuum chamber of the accelerator and the array of data files is recorded to the hard disc of the computer.

\section{Results}

The results of the four latest runs $(2010-2012)$ of the Nuclotron have demonstrated that the MCP-based detector makes it possible to measure the beam orbit, space-time characteristics, and the beam transport quality (beam losses during circulation).

Figure 5 shows the space-time beam structure obtained by the MCP detector (the upper plot). The evolution of the magnetic field of the accelerator is shown in the lower plot. It can be seen that as soon as the magnetic field reaches the "plateau" the beam orbit shifts toward the outer side of the accelerator chamber. Then the beam is set on the inner wall of the chamber by the growing field at the end of the "plateau".

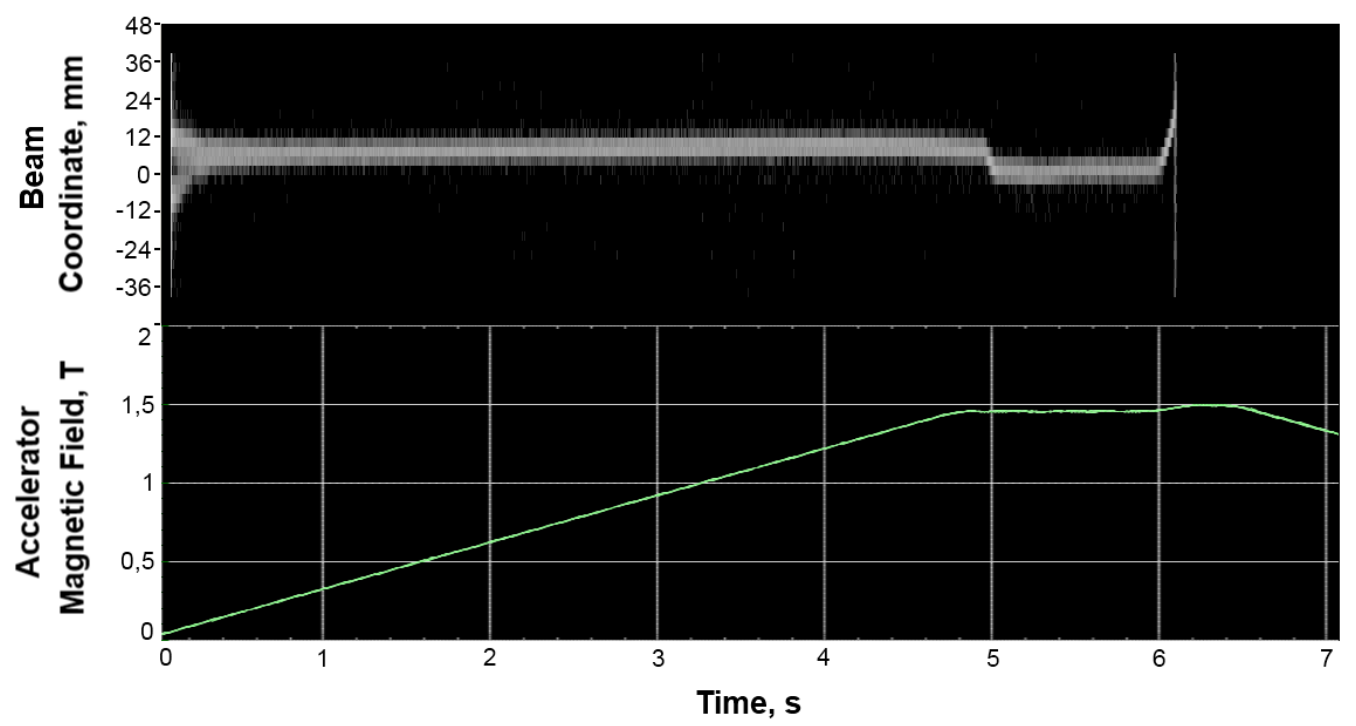

Figure 5: The data from the MCP detector (the upper plot) and the magnetic field of the accelerator (the lower plot).

For a detailed analysis of the beam structure we can vary the time interval value of measurements. Figure 6 shows the beam distribution with specified time intervals: the whole acceleration cycle; the injection moment and the beam extraction. 


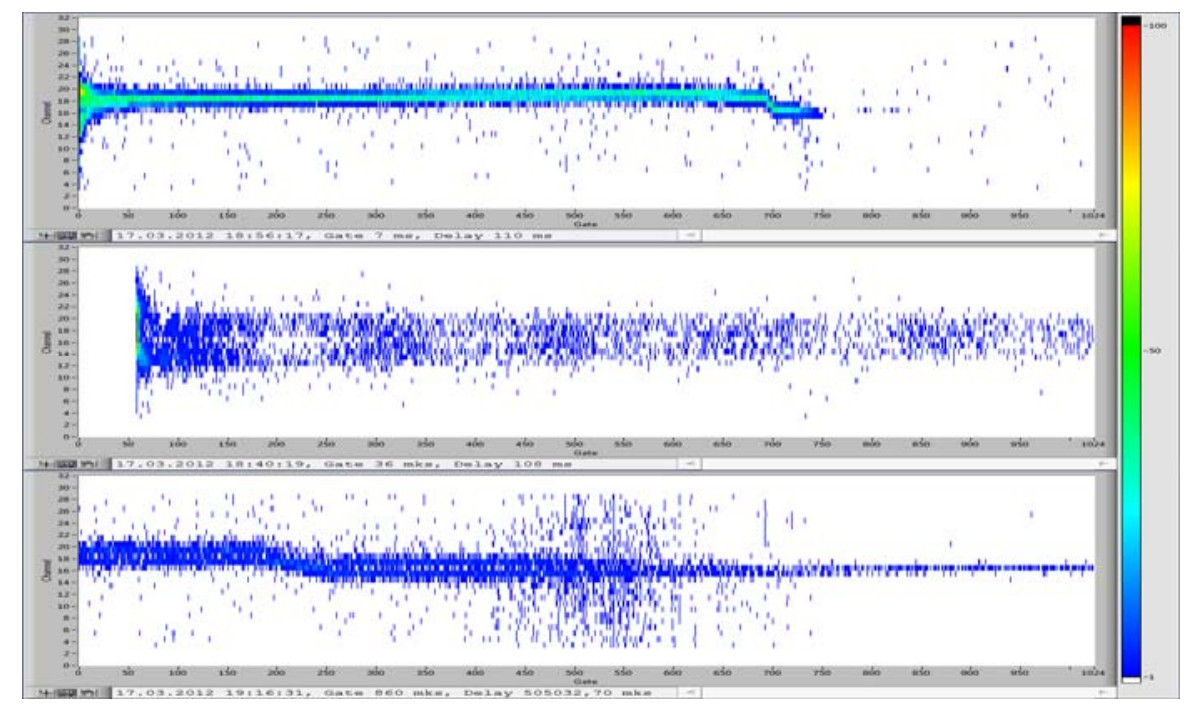

Figure 6: The beam space-time distribution at different values of time interval of measuring. The whole acceleration cycle is in the upper picture (the measurement time is $7.2 \mathrm{~s}$ ); the injection moment is in the middle picture $(37 \mathrm{~ms})$ and the beam extraction is in the lower picture $(0.9 \mathrm{~s})$.

The beam, circulating in the accelerator chamber, is partly or totally lost. The MCP-based monitoring system can register the time instant of the beam interaction with the accelerator chamber via typical "actuation" lines of all the detector channels. The accelerator cycles with partial beam losses are shown in Fig. 7 for two values of the cutoff electrode voltage of the detector.
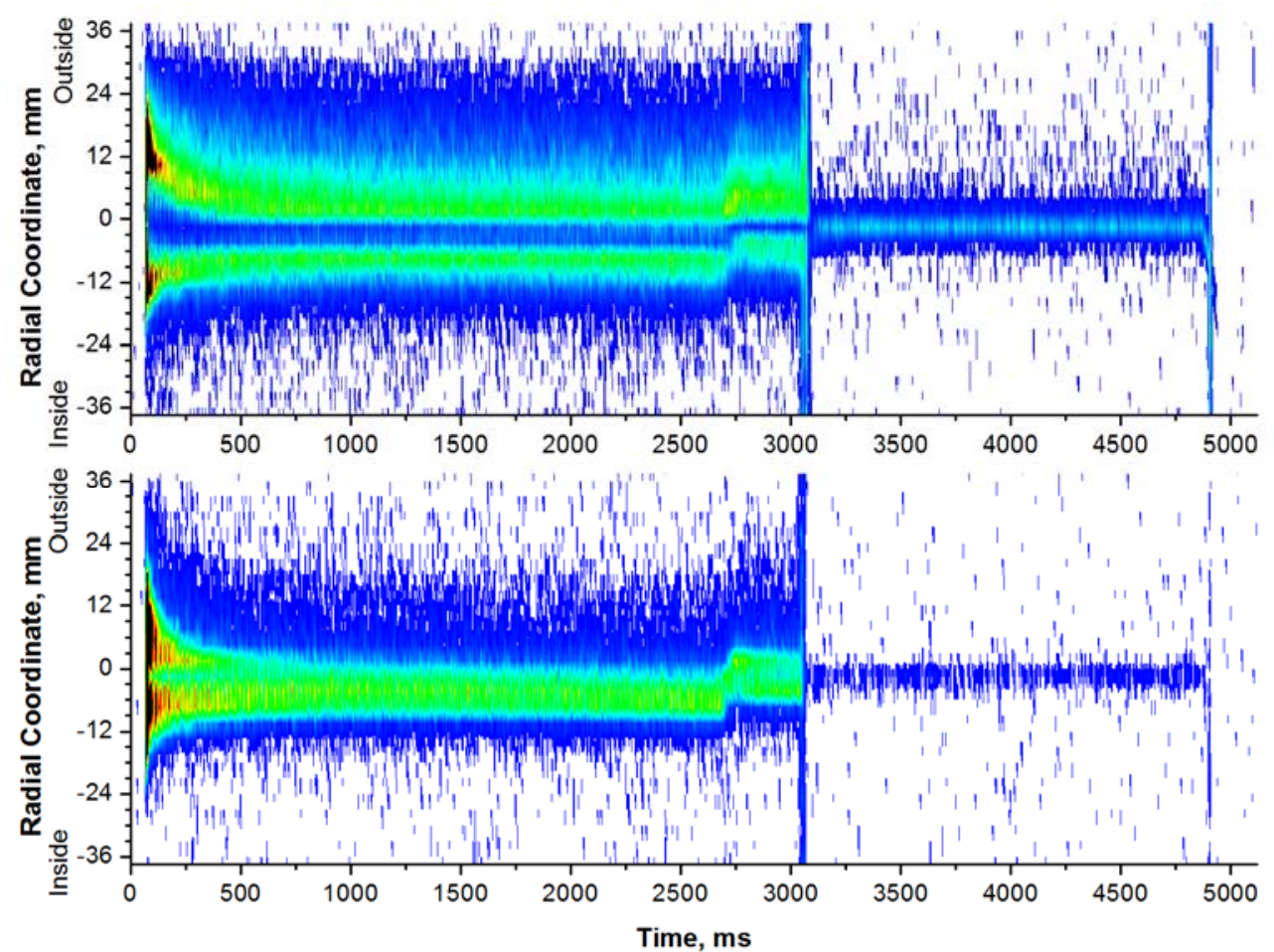

Figure 7: One acceleration cycle at two different voltages of the detector locking electrode. 
The actuation of all the detector channels at the time instant of the beam interaction with the accelerator chamber can be observed in both parts of Fig. 7 (vertical line after the "plateau"). After that the beam intensity drops in a step-like way. The detector overload in the time interval from injection to the intensity drop can be seen in the upper part of Fig. 7. The detector overload was overcome by optimization of the cutoff electrode voltage (see the lower part of Fig. 7).

\section{Conclusions}

The new diagnostic system of the Nuclotron circulating beam based on MCP allows one to study the spatial and temporal beam structure in the intensity range from $10^{5}$ to $10^{9}$ of singly charged ions.

The electrostatic system of the detector provides the optimal regime of the detector operation depending on the vacuum, the beam type and intensity, thus extending the working range of the detector with respect to intensities and types of accelerated ions.

The detector design makes it possible to change the width of the sensitive pads to $0.5 \mathrm{~mm}$ which determines the spatial resolution of the detector. At present, the sensitive area of the detector is $90 \mathrm{~mm}$ and the spatial resolution is $3 \mathrm{~mm}$.

The software for remote control of the detector parameters has been developed and tested; this software provides digital and graphical information to the control panel of the accelerator in the operator-friendly form.

This detector is efficient to adjust the time structure of slow extraction of the Nuclotron accelerated ion beams.

\section{References}

[1] V.A. Moskalev, G.I. Sergeev, Measurement of parameters of charged particle beams, Energoatomizdat, Moscow 1991.

[2] M.A. Gruntman, Coordinate-sensitive detectors based on microchannel plates (review), IET No.1 1984 .

[3] B.S. Ishkhanov, I.M. Kapitonov, E.I. Kebin, Particles and nuclei. Experiment, MSU Press, Moscow 2005.

[4] A.A. Baldin et al., Diagnostics system of the Circulating Beam of the Nuclotron Based on Microchannel Plates, JINR Rapid Communications No.2 1997.

[5] G.A. Feofilov et al., Position-sensitive MCP-based detectors with high timing resolution: some results and perspectives, Nuclear Instruments and Methods in Physics Research A 3671995.

[6] F.F. Valiev, Microchannel plates as a detector for $800 \mathrm{MeV/c}$ charged pions and protons, JINR Rapid Communication No.4 1991. 